\title{
1 An integrated modelling approach for flood simulation in the \\ 2 urbanized Qinhuai River basin, China
}

3

4 Runjie $\mathrm{Li}^{1,2}$, Jinkang $\mathrm{Du}^{1,2}$, Guodong Bian ${ }^{1,2}$, Yuefeng Wang ${ }^{3}$, Changchun Chen ${ }^{4}$, 5 Xueliang Zhang ${ }^{1,2}$, Maohua $\mathrm{Li}^{1,2}$, Shanshan Wang ${ }^{1,2}$, Senyao $\mathrm{Wu}^{1,2}$, Shunping $\mathrm{Xie}^{1,2}$,

6 Long Yang $^{1 *}$, Chong-Yu Xu ${ }^{5}$

7

$8 \quad{ }^{1}$ School of Geography and Ocean Science, Nanjing University, Nanjing, Jiangsu Province

9210023 , China.

$10 \quad{ }^{2}$ Jiangsu Center for Collaborative Innovation in Geographical Information Resource

11 Development and Application, Nanjing, Jiangsu Province 210023, China.

$12{ }^{3}$ School of Geography and Tourism, Chongqing Normal University, Chongqing 401331,

13 China

$14{ }^{4}$ School of Geographical Sciences, Nanjing University of Information Science and

15 Technology, Nanjing, Jiangsu Province 210044, China

$16{ }^{5}$ Department of Geosciences, University of Oslo, PO Box 1047 Blindern, N-0316 Oslo,

17 Norway.

18 *Corresponding author: E-mail address: yanglong@nju.edu.cn (L. Yang)

19 Declarations

20 Funding

21 This work was supported by the National Natural Science Foundation of China (No.

2241771029 and No. 41371044), the Research Council of Norway (FRINATEK Project 
23 274310) and the Strategic Priority Research Program of the Chinese Academy of

24 Sciences (No. XDA23040202)

\section{Conflicts of interest}

26 The authors declare that they have no conflicts of interest.

27 Availability of data and material

28 The data that support the findings of this study are available from the corresponding

29 author upon reasonable request.

30 Code availability

31 The code that supports the findings of this study is available from the corresponding

32 author upon reasonable request.

33 Authors' contributions

34 All authors contributed to the study conception and design. Material preparation, data

35 collection and analysis were performed by Runjie Li, Guodong Bian and Jinkang Du.

36 The first draft of the manuscript was written by Runjie Li, and all authors commented

37 on previous versions of the manuscript. The modified manuscript was completed by

38 Jinkang Du, Runjie Li and Long Yang. All authors read and approved the final

39 manuscript.

\section{Acknowledgements}

41 This work was supported by the National Natural Science Foundation of China (No. 4241771029 and No. 41371044), the Research Council of Norway (FRINATEK Project 43 274310), and the Strategic Priority Research Program of the Chinese Academy of 44 Sciences (No. XDA23040202), whose support was greatly appreciated. 
45 Abstract

46 The accurate simulation and prediction of flood response in urbanized basins remains

47 a great challenge due to the spatial and temporal heterogeneities in land surface properties. We hereby propose an integrated modelling approach that consists of a semidistributed conceptual hydrological model and a novel parameterization strategy. The modelling approach integrates the Xinanjiang (XAJ) model, Taihu Basin (TB) model,

51 and Nash instantaneous unit hydrograph (IUH) into a framework. Model parameters

52 are calibrated by optimizing their relationships with corresponding physical factors.

53 The proposed modelling approach is applied in the Qinhuai River basin (QRB), China.

54 The modelling approach shows satisfactory performance in flood simulation both for 55 calibration and validation of flood events in the QRB. The approach has temporal and 56 spatial prediction capability by using the established relationships between parameter 57 values and physical factors. Robustness analysis reveals that the different sets of flood events used for parameter relationship calibration led to similar model performance.

59 Numerical experiments show that impervious coverage poses strong influences on the 60 model performance and needs to be considered in flood routing simulations for small61 or medium-intensity flood events.

\section{Keywords}

63 Urbanization, Hydrological model, Model calibration, Flood response, Parameter 64 estimation 


\section{Introduction}

As one of the most extensive anthropogenic activities, urbanization has triggered a variety of environmental issues (Booth and Jackson 1997; Patra et al. 2018; Zhang et al. 2018), among which hydrological alterations have attracted increasing concern in the past several decades. Urban development increases impervious surface area and artificial drainage systems, which dramatically alter hydrological processes (Braud et al. 2013; Oudin et al. 2018; Schueler et al. 2009), such as an increase in surface runoff, a decrease in infiltration and changes in groundwater discharge (e.g., Burns et al. 2005; Salvadore et al. 2015). Previous studies have shown that disastrous flood events have become more frequent due to urbanization (Hu 2016; Hundecha and Bardossy 2004).

Hydrological modelling is the most useful and effective tool to examine the impacts of urbanization on hydrological processes (Jacobson 2011; Trinh and Chui 2013). Hydrological models can be typically divided into three categories: lumped, semi-distributed and distributed models (Arnold and Gibbons 1996; Bach et al. 2014; Salvadore et al. 2015). Of all three categories, semi-distributed models reasonably consider the spatial heterogeneity of subcatchments or hydrologic units compared with the lumped model. In addition, semi-distributed models are superior to distributed models in terms of reducing computational complexity and the number of parameters. Therefore, semi-distributed hydrological models are broadly employed in urban hydrological studies, e.g., the Storm Water Management Model (SWMM), Hydrologic Engineering Center-Hydrologic Modeling System (HEC-HMS), Soil and Water Assessment Tool (SWAT), and so on (e.g., Abbaspour et al. 2015; Arnold and Fohrer 
2005; Lee and Heaney 2003; Lhomme et al. 2004; McColl and Aggett 2007; Valeo and Moin 2000; Zhao 1992).

In this study, we propose a semi-distributed conceptual modelling approach that combines the Xinanjiang (XAJ) model (Zhao 1992), Taihu Basin (TB) model (Cheng et al. 2006), and Nash instantaneous unit hydrograph (IUH) method (Nash 1960). The modelling approach uses spatially variable parameters and adopts conceptual methods to calculate runoff generation and routing. It has relatively feasible parameterization and high computational efficiency. Model calibration based on the observed hydrological data is necessary for obtaining better model performance. To reduce the number of calibrated parameters for semi-distributed models, parameters with low sensitivities or direct physical meanings can be assigned to their 'typical' value from current literature or field measurements. For example, hydraulic properties of soil can be obtained from the literature and from field measurements (Refsgaard 1997; Rodriguez et al. 2008). The ratio and connectivity of impervious surfaces can be obtained using remote sensing products (Lee and Heaney 2003). Parameters with high sensitivities are calibrated based on observed data. However, the observed data are usually scarce and unavailable for the calibration of parameters in each sub-basin. To determine the values of some highly sensitive parameters (especially for process-related parameters), one solution is to use regression equations to calculate those parameters based on physical data as independent variables (Xu 1999, 2003; Yang et al. 2018). For instance, Bedient and Huber (1992) presented regression equations for determining the time of concentration and the storage coefficient of the Clark unit hydrograph for each 
sub-basin. These equations represent relationships between parameters and other easily

110 measurable physical factors, such as channel length, channel slope, and percentage of

111 developed land. However, parameter estimations using these relationships are not

112 always accurate because different basins or sub-basins may have different relationships;

113 thus, the estimated values might be used only as initial values of the parameters for

114 further calibration (USACE-HEC 2000). Ideally, these equations should be rebuilt or

115 calibrated for each individual sub-basin. However, it is almost impossible to rebuild the

116 equations due to the lack of observed data for each sub-basin. To address this problem,

117 a parameterization scheme was proposed in this study by directly building unified

118 equations to calculate parameters for each sub-basin, and the coefficients in the

119 equations can be calibrated based only on the streamflow data at the basin outlet. In this

120 way, the limitation of a lack of observation data for sub-basins can be solved, the

121 number of calibrated model parameters can be reduced, and the calibration efficiency

122 can be improved.

123 Therefore, the objectives of this study are to (1) propose a framework that uses a

124 semi-distributed rainfall-runoff model for simulating flood processes in a mesoscale

125 urbanized basin; (2) propose a parameterization scheme by establishing relationships

126 between model parameters and potential driving factors; and (3) evaluate the simulation

127 and prediction capacity of the integrated modelling approach.

129 The Qinhuai River basin (QRB) is located in Jiangsu Province, south-eastern China 
130 (Fig. 1). The drainage area is $2,631 \mathrm{~km}^{2}$. The peak rainfall from June to August often

131 leads to severe flood hazards. Fast urbanization further increases the frequency of

132 floods in this region (Du et al. 2013). The main land-use types include water surface,

133 paddy land, urban land, dry land, and woodland. There are seven reservoirs, four

134 hydrological stations and seven rain gauges in the QRB. The Qinhuaixinhe and

135 Wudingmen hydrological stations are located at the outlet of the basin (see Fig. 1 for

136 locations).

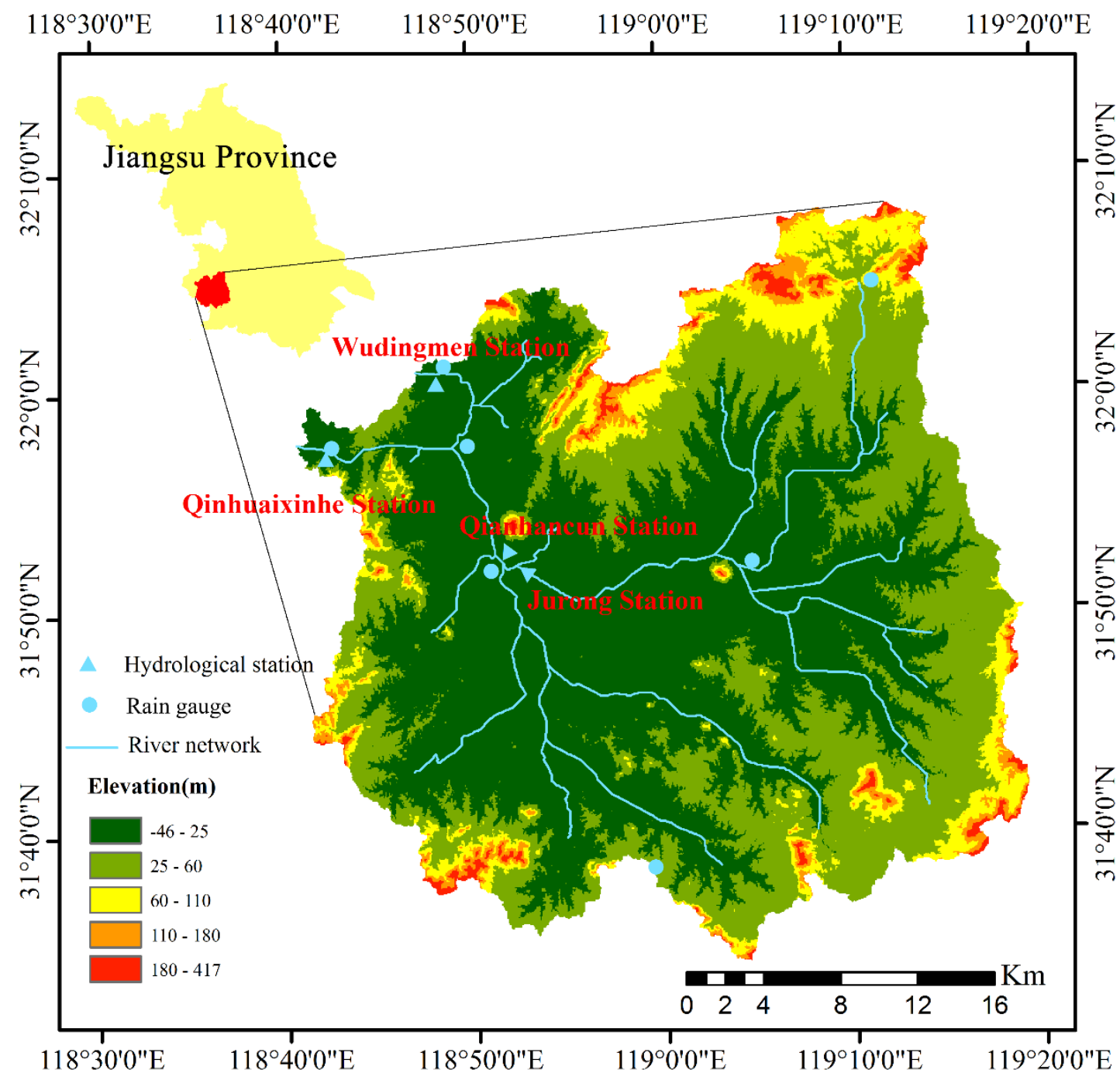

Fig. 1 Overview of the study area and location of hydrometeorological stations

139 Fourteen isolated flood events were selected from 1986 to 2015. The hourly 
140 rainfall data for the seven rain gauges, the hourly discharge data from Qianhancun

141 Station and Jurong Station, and the instant peak flow and daily discharge data from

142 Wudingmen Station and Qinhuaixinhe Station for the flood events were collected from

143 the Nanjing Hydrological Bureau. The Thiessen polygon approach was used to

144 interpolate rainfall. The hourly outflows of Wudingmen Station and Qinhuaixinhe

145 Station were obtained by linear interpolation of the instant peak flow and the daily

146 discharge of the stations. The summary of rainfall at the basin scale and runoff observed

147 at Qianhancun station in the fourteen flood events is given in Table 1.

148 Table 1 Summary of selected rainfall and runoff events

\begin{tabular}{|c|c|c|c|c|c|c|}
\hline \multirow[b]{2}{*}{ Storm no. } & \multirow[b]{2}{*}{ Storm date } & \multicolumn{3}{|c|}{ Basin-scale rainfall } & \multicolumn{2}{|c|}{$\begin{array}{l}\text { Streamflow at the Qianhancun } \\
\text { Station }\end{array}$} \\
\hline & & $\begin{array}{l}\text { Depth } \\
(\mathrm{mm})\end{array}$ & $\begin{array}{l}\text { Duration } \\
\text { (h) }\end{array}$ & $\begin{array}{l}\text { Average } \\
\text { intensity } \\
(\mathrm{mm} / \mathrm{h})\end{array}$ & $\begin{array}{l}\text { Peak } \\
\left(\mathbf{m}^{3} / \mathbf{s}\right)\end{array}$ & $\begin{array}{l}\text { Time to peak } \\
\text { (h) }\end{array}$ \\
\hline 198707 & July 2,1987 & 228.3 & 119 & 1.9 & 731 & 83 \\
\hline 199106 & June12,1991 & 333.9 & 115 & 2.9 & 964 & 81 \\
\hline 199607 & July 3, 1996 & 152.3 & 75 & 2.0 & 707 & 81 \\
\hline 200607 & July 19, 2006 & 171.4 & 91 & 1.9 & 513 & 79 \\
\hline 200808 & August 1,2008 & 115.3 & 32 & 3.6 & 654 & 37 \\
\hline 200907 & July 21, 2009 & 172.7 & 62 & 2.8 & 775 & 43 \\
\hline 201007 & July 12, 2010 & 153.2 & 32 & 4.8 & 491 & 34 \\
\hline 201106 & June 25, 2011 & 93.1 & 30 & 3.1 & 588 & 30 \\
\hline 201107 & July 18, 2011 & 95.6 & 56 & 1.7 & 517 & 30 \\
\hline 201207 & July 14,2012 & 62.8 & 17 & 3.7 & 380 & 20 \\
\hline 201208 & August 8,2012 & 78.3 & 37 & 2.1 & 667 & 47 \\
\hline 201307 & July 5,2013 & 125.9 & 62 & 2.0 & 497 & 37 \\
\hline 201407 & July 4, 2014 & 99.6 & 29 & 3.4 & 772 & 35 \\
\hline 201506 & June 16,2015 & 179.1 & 43 & 4.2 & 939 & 44 \\
\hline
\end{tabular}

149 Fig. 2 shows changes in land use/land cover in the QRB during the period 1988- 
150 2015. The land use/land cover was extracted from Landsat satellite images based on the

151 rotation forest classifier method (Rodriguez et al. 2006; Bian et al. 2017). A noticeable

152 change was found in the increase in impervious coverage from $3.92 \%$ to $19.76 \%$, which

153 was caused by the decrease in paddy field (from $50.09 \%$ to $29.94 \%$ ) during the same 154 period.
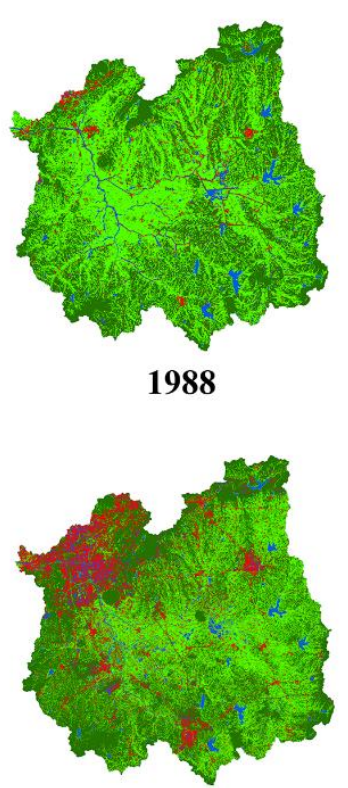

2006

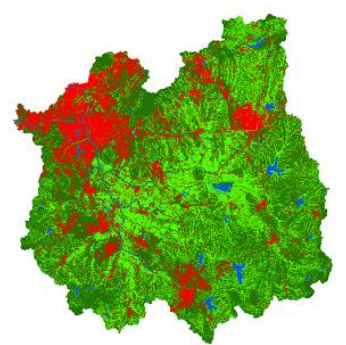

2013

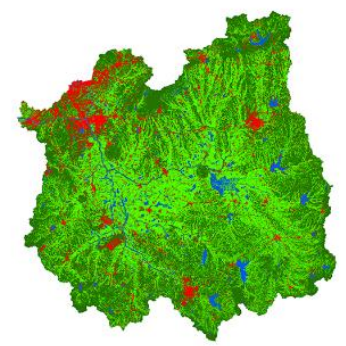

2001

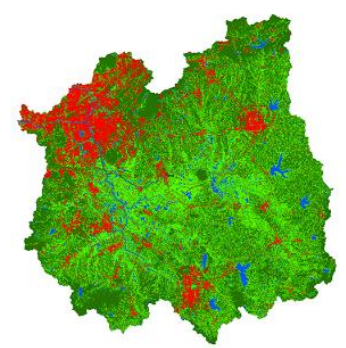

2009

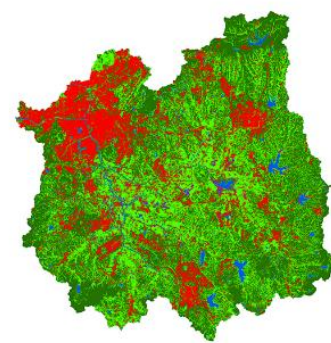

2015
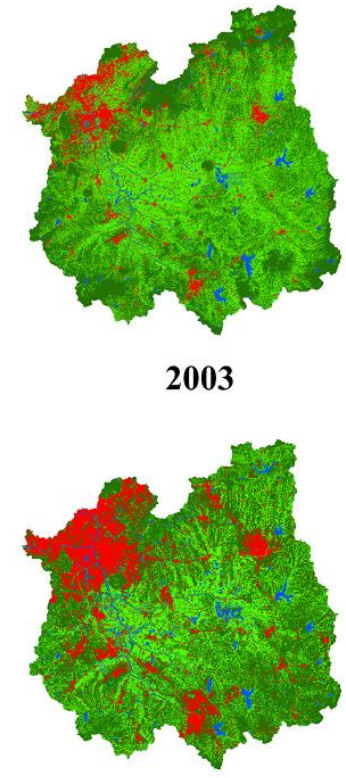

2011
155

156

Fig. 2 Land use classification results of the Qinhuai River basin

\section{Methodology}

\subsection{Overview of the modelling framework}

159 The semi-distributed model has three individual modules: (i) runoff generation, (ii) 
161 the XAJ model and TB model; the runoff separation module is mainly adopted from the

162 XAJ model; and the runoff routing module is established based on the XAJ model using

163 the Nash IUH method (Nash 1957, 1960). The structure of the semi-distributed model

164 is given in Fig. 3. The key feature of the XAJ model is that runoff is generated and

165 calculated only when the soil moisture content reaches field capacity (Zhao et al. 1980).

166 The TB model is a hydrological modelling system that considers the heterogeneity in

167 runoff generation by categorizing land surfaces into four main categories, i.e., water

168 surface, urban, paddy field, and non-irrigated farmland (Cheng et al. 2006). The QRB

169 is divided into 18 sub-basins. The three modules were established for each of the sub-

170 basins.

171

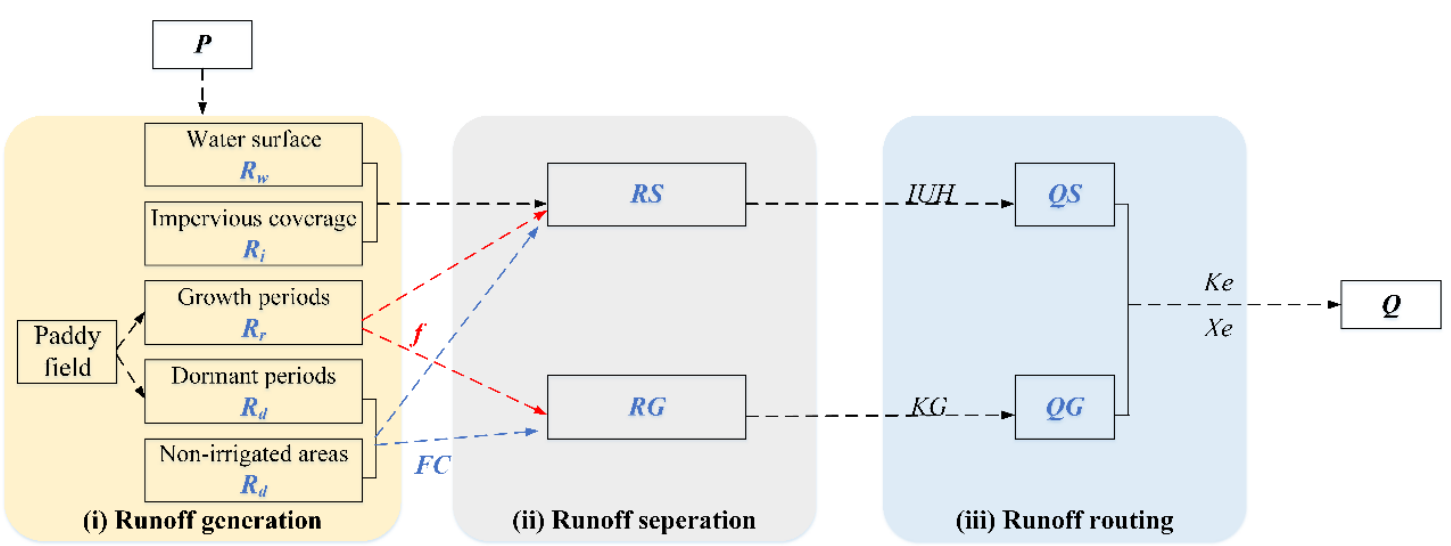

Fig. 3 The structure of the semi-distributed hydrological model

\subsubsection{Runoff generation}

174 In the runoff generation module, four types of land uses are considered: water surface,

175 paddy field, impervious coverage, and non-irrigated areas.

176 The runoff generated from the water surface at each time interval is calculated as: 


$$
R_{w}=P-\beta \times E_{p}
$$

178 where $R_{w}$ is the runoff generated from the water surface $(\mathrm{mm}), P$ denotes the

179 precipitation $(\mathrm{mm}), E_{p}$ denotes the potential evapotranspiration $(\mathrm{mm})$, and $\beta$ denotes the 180 adjustment factor of $E_{p}(-)$.

181 Paddy fields can be divided into dormant periods and rice-growing periods. The 182 runoff generated from paddy fields is calculated in different rice-growing periods and 183 at each time interval, and it is described as:

$$
H_{2}=P-\alpha \times E_{p}+H_{1}-f
$$

When $H_{2}>H_{p}$,

$$
R_{\mathrm{r}}=H_{2}-H_{d}, H_{1}=H_{d}
$$

193 where $R_{r}$ is the runoff generated from the paddy field in the rice-growing period (mm)

194 at each time interval, $a$ represents the water requirement coefficient of the paddy field

$195(-), f$ is the infiltration in the paddy field (mm) at each time interval, and $H_{1}$ and $H_{2}$ are

196 the depths of water in the paddy field at the beginning and end of each time interval 197 (mm), respectively. $H_{p}$ represents the depth of submergence tolerance (mm). $H_{u}$ and $H_{d}$ 198 denote the suitable top and bottom depths of water needed at different growing periods 
199

$(\mathrm{mm})$, respectively.

The runoff generated from impervious coverage at each time interval is calculated as:

$$
R_{\mathrm{i}}=\varphi \times P
$$

where $R_{i}$ is the runoff generated from impervious coverage $(\mathrm{mm})$, and $\varphi$ is the runoff coefficient (-).

The runoff generated from non-irrigated areas and dormant paddy fields at each time interval is estimated based on the XAJ model. In the saturated area where the soil moisture content reaches field capacity, the runoff is calculated using Eq. (8). Otherwise, the runoff calculation can be found by referring to Zhao (1992).

$$
R_{\mathrm{d}}=P-\left(W M-W_{o}\right)-E
$$

where $R_{d}$ represents the runoff generation $(\mathrm{mm})$ in the time interval, $W M$ is the areal mean tension water capacity $(\mathrm{mm}), W_{0}$ is the initial soil water $(\mathrm{mm})$, and $E$ is the actual evapotranspiration (mm). Evapotranspiration is not considered in the runoff generation module due to its negligible contributions to flood simulation.

215 Runoff separation aims to divide the generated runoff into two or more components

216 according to land-use type. All the runoff generated from the water surface and

217 impervious coverage would turn to surface runoff. The runoff from non-irrigated areas 218 and dormant paddy fields is subdivided into surface runoff and groundwater runoff 219 based on the free water capacity distribution curve ( $\mathrm{Li}$ et al. 2018; Meng et al. 2016; 
220 Zhao 1992), and the runoff in paddy fields calculated from Eqs. (2) to (6) turns to

221 surface runoff; finally, infiltration at a steady rate contributes to groundwater runoff.

222 For each sub-basin, the total surface runoff (groundwater runoff) is an area-weighted

223 summation of runoff from the four land-use types (paddy land and non-irrigated areas).

\section{$224 \quad 3.1 .3$ Runoff routing}

225 The surface runoff is routed directly to the outlet of each sub-basin by the Nash IUH 226 method (Nash 1957, 1960), while the groundwater runoff is routed using the linear 227 reservoirs method (Zhao et al. 1980; Zhao 1992). The discharge from the upper sub228 basins is routed through the river network to the outlet of the sub-basin by the 229 Muskingum successive-reaches model (Deng et al. 2009). The outflow at the outlet of 230 each sub-basin is the summation of the surface runoff and groundwater discharge of the 231 sub-basin and the river network routing discharge from the upper sub-basins.

\section{$232 \quad 3.1 .4$ Reservoir operation}

233 Reservoirs can temporarily store flood water and release it later, which effectively 234 lowers the magnitude and frequency of floods in downstream reaches. The changes in 235 reservoir volume are simulated by the storage function approach as:

$$
\frac{d V}{d t}=I N F-O F
$$

237 where $V$ is the reservoir storage $\left(\mathrm{m}^{3}\right), t$ is the time $(\mathrm{s}), I N F$ is the inflow $\left(\mathrm{m}^{3} / \mathrm{s}\right)$, and $O F$ 238 is the release $\left(\mathrm{m}^{3} / \mathrm{s}\right)$. The details on reservoir operation can be found in Du et al. (2016). 


\subsection{Model calibration strategy}

240 To reduce the number of calibrated parameters and improve the calculation efficiency

241 of the model, the following parameterization strategy is proposed. (1) Parameters with

242 low sensitivities or direct physical meanings are set to their 'typical' values based on a

243 literature review and expert experience. (2) Parameters with high sensitivity are

244 estimated based on the proposed parameterization scheme: calibrating relationships

245 between parameters and influencing factors, such as urbanization index (impervious

246 ratio) and basin characteristics (i.e., area, slope and length). (3) Other parameters are

247 determined through calibration.

248 The parameters with high sensitivity and the typical values of most parameters in

249 the proposed model can be found in the literature (e.g., Li et al. 2018; Lin et al. 2011;

250 Meng et al. 2016; Zhao et al. 1980; Zhao 1992). For the parameters with high sensitivity

251 for groundwater routing, the Muskingum successive-reaches method and the runoff

252 coefficient of the impervious surface are manually optimized based on the trial-and-

253 error method. The parameters of the Nash IUH method for surface runoff routing for

254 each sub-basin are calibrated by using the proposed parameterization scheme. To reduce

255 parameter dimensions, we assume that the Nash IUH parameters of all sub-basins have

256 the same functional relationships with sub-basin characteristics (e.g., area, slope and

257 length of the river network) and urbanization index (i.e., impervious rate), and the

258 relationships between the Nash parameters, urbanization index and basin characteristics

259 can be expressed as follows:

$$
n=f_{1}(A, S L, L E, I M, \ldots)
$$


262 where $f_{1}$ and $f_{2}$ are functions; $n$ and $k$ are the number and storage coefficient of linear

263 reservoirs of the Nash IUH, respectively; and $A, S L, L E$, and $I M$ denote the 264 characteristics of the area, slope, length, and impervious ratio of a sub-basin, 265 respectively. The Nash IUH parameters of each sub-basin can be calculated based on 266 the relationships. Parameter optimization thus turns into the optimization of functions 267 (10) and (11). The best mathematical forms of relationships $f_{1}$ and $f_{2}$ could be obtained 268 by maximizing the average NSE for all calibrated flood events. The enumeration 269 optimal method or other optimal methods could be implemented to find the optimal 270 parameters for each relationship by maximizing the average NSE. The proposed 271 parameterization strategy can improve model calibration efficiency by reducing the 272 number of calibrated parameters.

$273 \quad 3.3$ Temporal and spatial prediction capabilities of the integrated modelling 274 approach

275 To test the temporal prediction capability of the approach, six flood events from 1987 276 to 2009 were used for the relationship calibration, while eight flood events from 2010 277 to 2015 were used for model validation. Flood records from Qianhancun Station were 278 used. The proxy-basin test was performed to verify the spatial prediction capability of 279 the approach, i.e., calibrate flood events on one catchment and validate them on another 280 catchment. The relationships of Nash IUH parameters were calibrated using the 281 discharge data for fourteen flood events at Qianhancun Station. The calibrated 
relationships were then used to predict flood events for the entire QRB.

283

284

285

286 287 calculated as follows:

288

\subsection{Evaluation criteria}

Four criteria were employed to evaluate the model performance (McCuen et al. 2006): the Nash-Sutcliffe efficiency (NSE), the coefficient of determination $\left(\mathrm{R}^{2}\right)$, the relative error of peak discharge $\left(D_{\mathrm{p}}\right)$ and the relative error of runoff volumes $\left(D_{\mathrm{v}}\right)$, and they are

292 where $Q_{c}(i)$ and $Q_{o}(i)$ denote the estimated and observed discharges for time period $i$

$293\left(\mathrm{~m}^{3} / \mathrm{s}\right)$, respectively; $Q_{c}$ and $Q_{o}$ represent the estimated and observed mean values $\left(\mathrm{m}^{3} / \mathrm{s}\right)$,

294 respectively; $n$ is the total number of observed discharges; and $Q_{p, c}$ and $Q_{p, o}$ are the peak

295 discharges of the estimated and observed hydrographs $\left(\mathrm{m}^{3} / \mathrm{s}\right)$, respectively.

\section{Results and Discussion}

\subsection{Model calibration and validation}




\subsubsection{Calibration of model parameters}

299 The runoff generation parameters over paddy land were set to suggested values and are

300 shown in Table 2 (Cheng et al. 2006). The parameters depend on different paddy

301 growing periods. The daily infiltration capacity was set to $1 \mathrm{~mm}$ due to the high

302 groundwater level and saturated soil during the growing season. The runoff coefficient

303 of impervious coverage was set to $0.65(-)$. The parameters $W M$ and $W_{0}$ for non-irrigated

304 areas were set to $120(\mathrm{~mm})$ and $30(\mathrm{~mm})$, respectively. The daily recession coefficient

305 of groundwater in the linear reservoir method was set to 0.9, and the Muskingum time

306 constant and weighting factor were calibrated to be $2(\mathrm{~h})$ and 0.2 , respectively. The best

307 relationships of the Nash IUH model parameters for all sub-basins were obtained by

308 maximizing the average NSE of calibrated flood events using the optimal enumeration

309 method:

$$
n=0.6 \times A^{0.05} \times I M^{-0.01}
$$

$$
k=1.0 \times A^{0.27} \times I M^{-0.2}
$$

312 where $n$ and $k$ are the number and storage coefficient of the Nash IUH of a selected sub-

313 basin, $A$ is the area of the sub-basin, and $I M$ is the impervious ratio of the sub-basin.

314 Table 2 The runoff generation parameters of paddy land from Cheng et al. (2006)

\begin{tabular}{cccccc}
\hline Duration (day) & $\begin{array}{c}\text { Depth of } \\
\text { submergence } \\
\text { tolerance (mm) }\end{array}$ & $\begin{array}{c}\text { Top suitable } \\
\text { water depth } \\
(\mathbf{m m})\end{array}$ & $\begin{array}{c}\text { Bottom } \\
\text { suitable } \\
\text { water depth } \\
(\mathbf{m m})\end{array}$ & $\begin{array}{c}\text { Coefficient of } \\
\text { water } \\
\text { requirement (-) }\end{array}$ & $\begin{array}{c}\text { Daily } \\
\text { infiltration } \\
\text { capacity (mm) }\end{array}$ \\
\hline $5.16 \sim 5.25$ & 20 & 10 & 5 & 1.00 & 1 \\
$5.26 \sim 6.23$ & 30 & 20 & 10 & 1.00 & 1 \\
$6.24 \sim 6.30$ & 50 & 30 & 20 & 1.35 & 1 \\
$7.1 \sim 8.4$ & 50 & 30 & 20 & 1.30 & 1 \\
$8.5 \sim 9.3$ & 50 & 40 & 30 & 1.65 & 1 \\
$9.4 \sim 9.16$ & 50 & 30 & 20 & 1.76 & 1 \\
\hline
\end{tabular}




$\begin{array}{llllll}9.17 \sim 10.20 & 20 & 10 & 0 & 1.50 & 1\end{array}$

\subsubsection{Model performance}

316 Table 3 indicates that the model achieves satisfactory performance during both

317 calibration and validation periods, with the average values of $\mathrm{R}^{2}$ and NSE exceeding

3180.9 and the average values of $D_{p}$ and $D_{v}$ being lower than 5\%. Fig. 4 demonstrates that

319 the estimated results are well synchronized with the observed hydrographs in terms of

320 both peak magnitudes and timing, indicating that the established model is applicable

321 for flood simulation in the QRB. The parameterization strategy of calibrating

322 relationships between model parameters and basin physical characteristics is also

323 effective and suitable for the study basin.

324 Table 3 The statistics of the model calibration and validation results at Qianhancun Station

\begin{tabular}{|c|c|c|c|c|c|c|}
\hline \multirow{2}{*}{ Period } & \multirow{2}{*}{ Flood code } & \multirow{2}{*}{$\begin{array}{c}\text { Land-use } \\
\text { pattern }\end{array}$} & \multicolumn{4}{|c|}{ Evaluation criteria } \\
\hline & & & $\mathbf{R}^{2}$ & NSE & $D_{P}(\%)$ & $D_{v}(\%)$ \\
\hline \multirow{7}{*}{ Calibration } & 198707 & 1988 & 0.91 & 0.91 & 4.65 & 1.24 \\
\hline & 199607 & 2001 & 0.86 & 0.84 & 12.84 & 5.75 \\
\hline & 200808 & 2009 & 0.92 & 0.90 & 2.44 & 7.84 \\
\hline & 201106 & 2011 & 0.95 & 0.95 & 3.74 & 1.15 \\
\hline & 201207 & 2013 & 0.93 & 0.92 & 1.84 & 4.42 \\
\hline & 201407 & 2015 & 0.93 & 0.93 & 2.14 & 3.85 \\
\hline & \multicolumn{2}{|c|}{ Mean value } & 0.92 & 0.91 & 4.61 & 4.04 \\
\hline \multirow{9}{*}{ Validation } & 199106 & 1988 & 0.92 & 0.91 & 0.90 & 3.51 \\
\hline & 200607 & 2006 & 0.88 & 0.87 & 1.96 & 4.00 \\
\hline & 200907 & 2009 & 0.97 & 0.94 & 2.34 & 9.29 \\
\hline & 201007 & 2011 & 0.96 & 0.95 & 2.05 & 4.39 \\
\hline & 201107 & 2011 & 0.77 & 0.76 & 1.90 & 4.04 \\
\hline & 201208 & 2013 & 0.97 & 0.96 & 5.43 & 3.84 \\
\hline & 201307 & 2013 & 0.97 & 0.96 & 1.43 & 4.94 \\
\hline & 201506 & 2015 & 0.97 & 0.97 & 1.17 & 3.42 \\
\hline & \multicolumn{2}{|c|}{ Mean value } & 0.93 & 0.92 & 2.15 & 4.68 \\
\hline
\end{tabular}




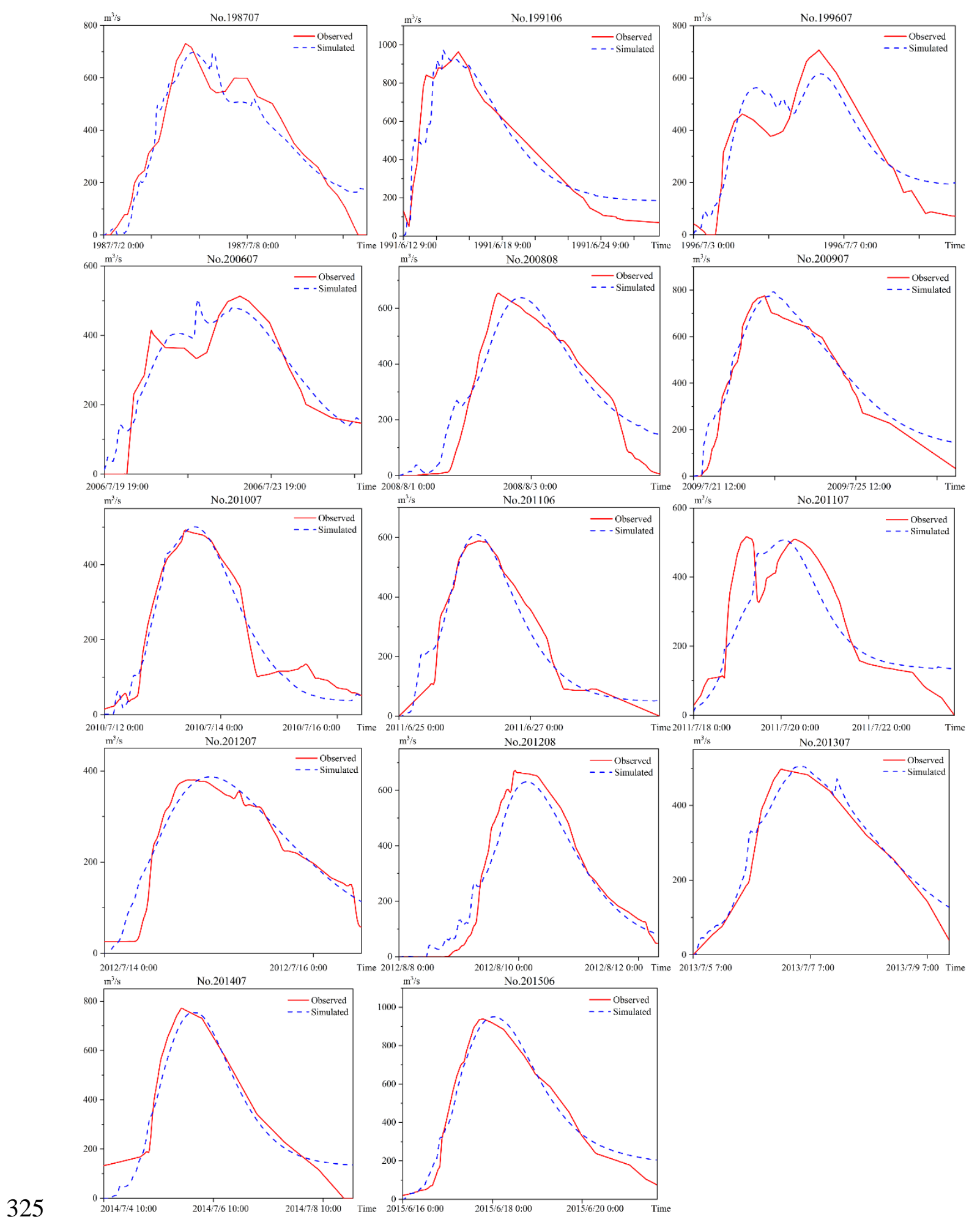

Fig. 4 Observed and simulated hydrographs of 14 floods at Qianhancun Station

Table 4 shows the temporal prediction results at Qianhancun Station. The average

328 values of $\mathrm{R}^{2}$ and NSE of all predictive flood events exceeded 0.9 , while the average values of $D_{p}$ and $D_{v}$ were less than $5 \%$, indicating that the proposed modelling approach 
330 can achieve good temporal prediction capacity. It also demonstrates that the

331 relationships calibrated from the early period can be used for later or future flood event

332 prediction.

333 The statistics of the spatial prediction performance of the approach are shown in

334 Table 4. The results indicate satisfactory prediction results in terms of simulating flood

335 events in the whole QRB, with the average $\mathrm{R}^{2}$ and NSE exceeding 0.85 and the average

336 values of $D_{p}$ and $D_{v}$ being lower than $10 \%$, which demonstrate that the calibrated

337 relationships over the upper and middle sub-basins can be transferred to the whole basin

338 for flood simulation.

339 Table 4 The statistics of model temporal prediction results at Qianhancun Station and spatial 340 prediction results in the whole QRB

\begin{tabular}{|c|c|c|c|c|c|c|}
\hline \multirow{2}{*}{$\begin{array}{l}\text { Prediction } \\
\text { capability }\end{array}$} & \multirow[t]{2}{*}{ Flood code } & \multirow{2}{*}{$\begin{array}{c}\text { Land-use } \\
\text { pattern }\end{array}$} & \multicolumn{4}{|c|}{ Evaluation criteria } \\
\hline & & & $\mathbf{R}^{2}$ & NSE & $D_{P}(\%)$ & $D_{v}(\%)$ \\
\hline \multirow{9}{*}{$\begin{array}{c}\text { Temporal } \\
\text { prediction } \\
\text { results }\end{array}$} & 201007 & 2011 & 0.94 & 0.94 & 0.82 & 1.26 \\
\hline & 201106 & 2011 & 0.97 & 0.96 & 4.58 & 3.70 \\
\hline & 201107 & 2011 & 0.78 & 0.77 & 2.10 & 3.60 \\
\hline & 201207 & 2013 & 0.93 & 0.93 & 6.08 & 3.24 \\
\hline & 201208 & 2013 & 0.98 & 0.98 & 1.37 & 2.05 \\
\hline & 201307 & 2013 & 0.97 & 0.96 & 5.04 & 7.11 \\
\hline & 201407 & 2015 & 0.94 & 0.93 & 3.38 & 2.31 \\
\hline & 201506 & 2015 & 0.98 & 0.97 & 1.36 & 4.43 \\
\hline & \multicolumn{2}{|c|}{ Mean value } & 0.94 & 0.93 & 3.09 & 3.46 \\
\hline \multirow{13}{*}{$\begin{array}{c}\text { Spatial } \\
\text { prediction } \\
\text { results }\end{array}$} & 198707 & 1988 & 0.94 & 0.94 & 3.76 & 2.03 \\
\hline & 199106 & 1988 & 0.93 & 0.93 & 1.76 & 2.62 \\
\hline & 199607 & 2001 & 0.84 & 0.82 & 4.88 & 7.27 \\
\hline & 200607 & 2006 & 0.96 & 0.95 & 3.70 & 4.76 \\
\hline & 200808 & 2009 & 0.92 & 0.92 & 6.73 & 4.24 \\
\hline & 200907 & 2009 & 0.96 & 0.94 & 10.81 & 10.53 \\
\hline & 201007 & 2011 & 0.96 & 0.96 & 13.72 & 1.47 \\
\hline & 201106 & 2011 & 0.96 & 0.89 & 11.04 & 18.44 \\
\hline & 201107 & 2011 & 0.85 & 0.79 & 10.85 & 15.00 \\
\hline & 201207 & 2013 & 0.91 & 0.9 & 10.98 & 0.66 \\
\hline & 201208 & 2013 & 0.96 & 0.99 & 1.22 & 8.68 \\
\hline & 201307 & 2013 & 0.94 & 0.91 & 5.92 & 8.22 \\
\hline & 201407 & 2015 & 0.89 & 0.76 & 19.72 & 19.82 \\
\hline
\end{tabular}




\begin{tabular}{ccccc}
\hline 201506 & 0.82 & 0.78 & 14.09 & 0.88 \\
Mean value & 0.92 & 0.89 & 8.51 & 7.47 \\
\hline
\end{tabular}

3414.2 Impacts of building relationships with or without the consideration of

342 impervious areas on model performance

343 The temporal and spatial variations of imperviousness should be considered in

344 hydrological modelling for urbanized basins due to the role of impervious surface in

345 influencing hydrological processes (Jacobson 2011; Praskievicz and Chang 2009).

346 Previous studies have shown that an increase in impervious areas had large effects on

347 the hydrological response for medium and small flood events but only small effects on

348 extreme events (Braud et al. 2013; Kaspersen et al. 2015). To further examine the effects

349 of impervious coverage on runoff generation and routing for flood events, the following

350 test was conducted to determine the impacts of establishing the relationships of Nash

351 IUH parameters with or without the consideration of imperviousness on model

352 performances. Three scenarios were considered in the test: (1) flood simulation scenario;

353 (2) temporal prediction scenario; and (3) spatial prediction scenario. The selected flood

354 events for calibration and validation/prediction were the same as those described in

355 Section 3. 
(1) Flood simulation scenario (2) Temporal prediction scenario (3) Spatial prediction scenario
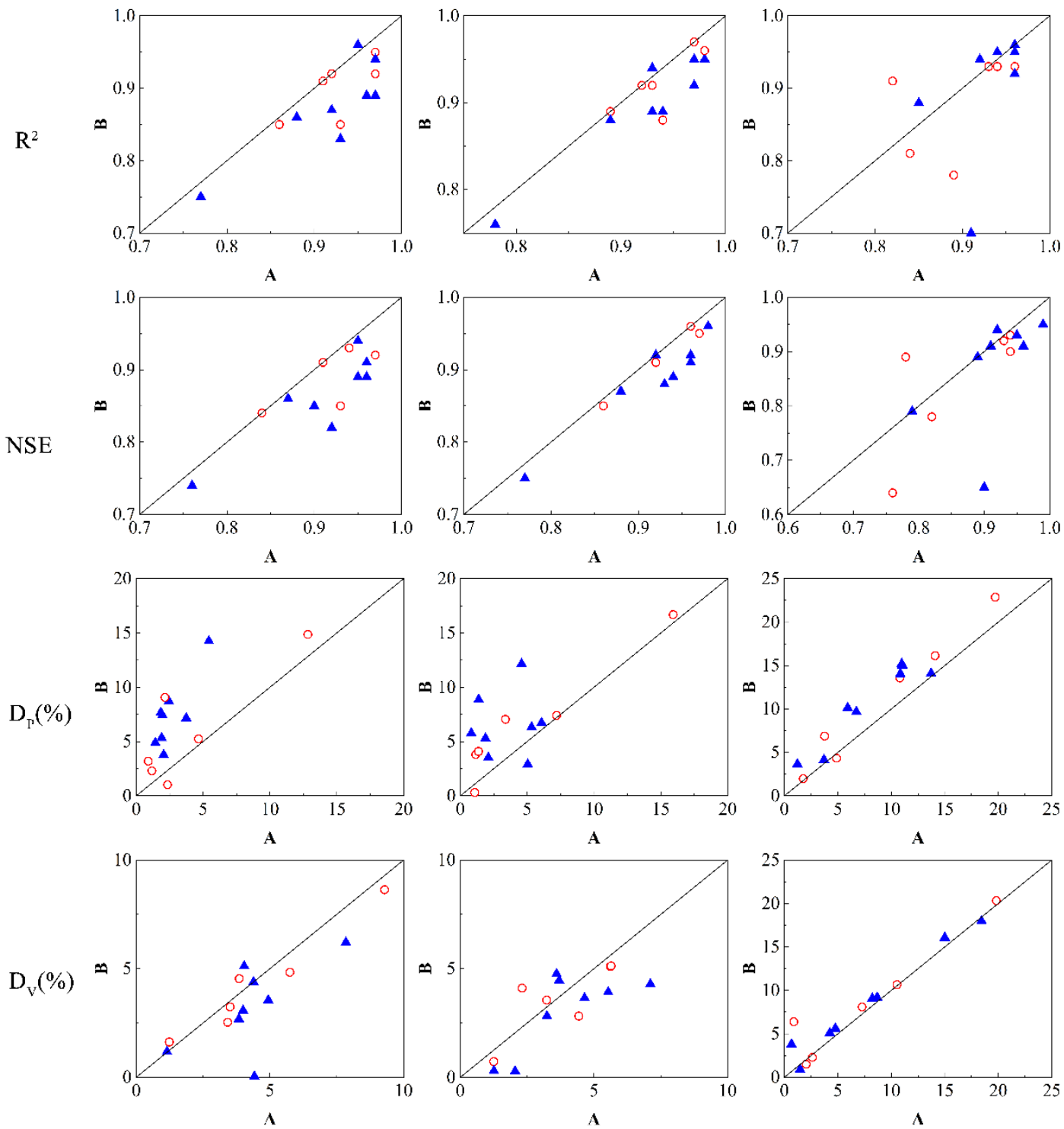

Fig. 5 Comparison of model simulation results, temporal prediction results and spatial prediction results with (A) and without (B) the consideration of imperviousness. Blue triangles represent small and medium floods (peak discharge lower than $700 \mathrm{~m}^{3} / \mathrm{s}$ ), red circles represent large floods (peak discharge higher than $700 \mathrm{~m}^{3} / \mathrm{s}$ )

As seen from Fig. 5, almost all $\mathrm{R}^{2}$ and NSE values for floods without the consideration of imperviousness in the surface runoff calculation are smaller than those that consider imperviousness, while the values of $D_{p}$ that consider imperviousness are obviously smaller than those that do not consider imperviousness. For $\mathrm{D}_{\mathrm{v}}$ values, all 
points are located near the 1:1 line, indicating that there is no distinct difference. This result is because impervious coverage only changes runoff-routing speed but not runoff

367 volume. The volume of runoff generation remains the same for surface runoff routing 368 regardless of whether imperviousness is considered.

369 For simulation and temporal prediction scenarios (Fig. 5), the values of $\mathrm{R}^{2}$, NSE, 370 and $\mathrm{D}_{\mathrm{p}}$ for small and medium floods were considerably improved when imperviousness

371 was considered compared to those that did not consider imperviousness, indicating that

372 imperviousness has a pronounced impact on model simulation for small and medium

373 flood events. For the spatial prediction scenario in the whole QRB (Fig. 5), the

374 improvements in the $\mathrm{R}^{2}$, NSE, and $\mathrm{D}_{\mathrm{p}}$ values for most flood events were not as obvious

375 as those in the first and second columns, which was likely because the impact of 376 urbanization on the surface runoff process has been relatively weakened with the 377 increase in basin size.

$378 \quad 4.3$ Impacts of flood event selection for calibrating the relationships on model 379 performance

380 The effects of using different flood events for calibrating relationships of Nash IUH 381 parameters on model performance were also analysed. Three scenarios were built: (1)

382 flood simulation scenario: six flood events different from the ones in Subsection 4.1 383 were selected for relationship calibration, the others were used for validation, and the 384 calibration and validation results were compared with those in Subsection 4.1; (2) 385 temporal prediction scenario: three flood events from 1987 to 1996 were used for 
386 calibration and the others were used for validation, the results were compared with 387 those calibrated by six flood events from 1987 to 2009 in Subsection 4.1; and (3) spatial 388 prediction scenario: the prediction results of the whole basin using relationships 389 calibrated by Qianhancun Station were compared with those calibrated by Jurong 390 Station.

(1) Flood simulation scenario (2) Temporal prediction scenario (3) Spatial prediction scenario
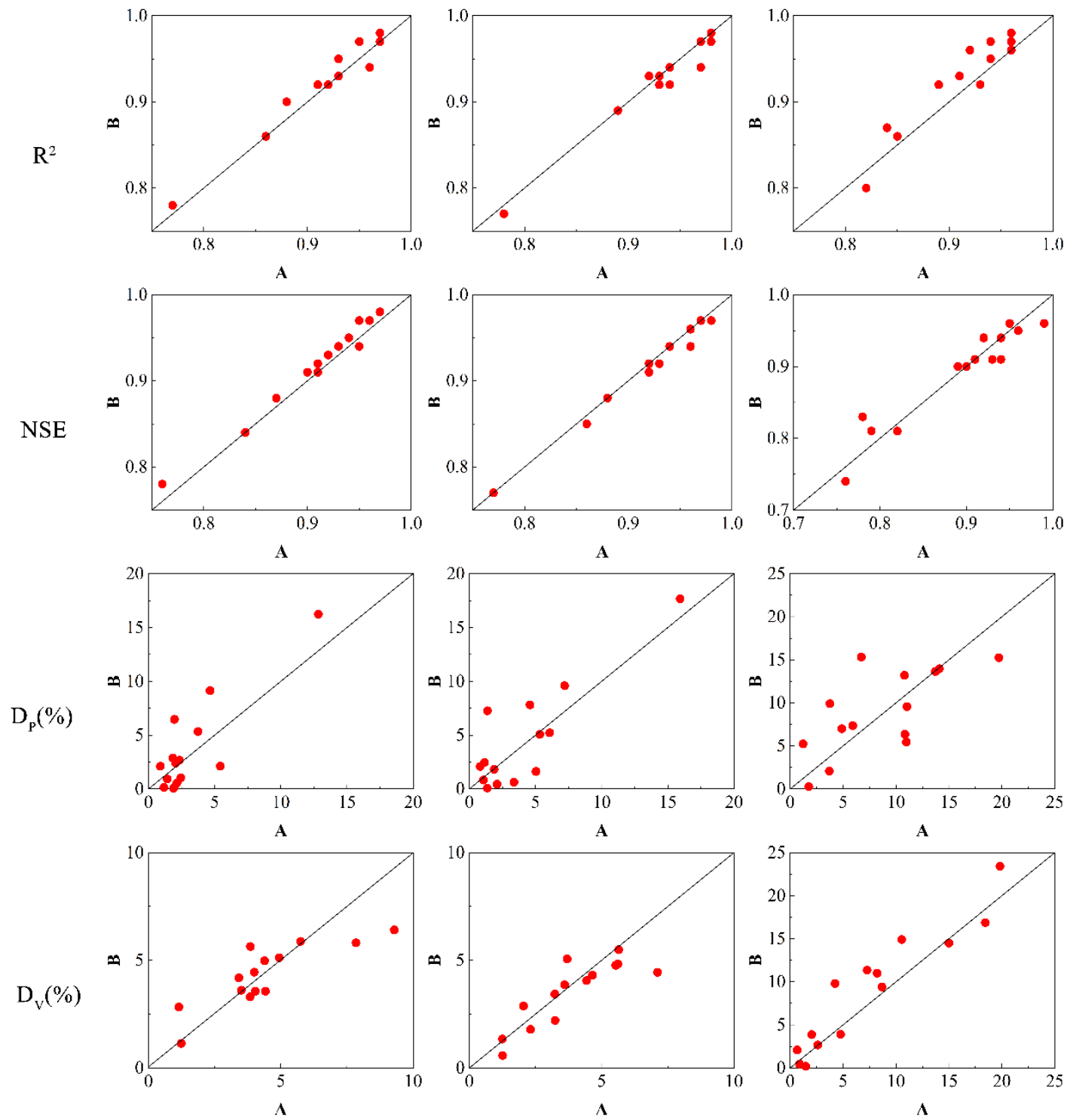

Fig. 6 Results of impacts of flood event selection for calibrating relationships on model performance. (1) Flood simulation scenario: comparison of calibration and validation results by selecting different flood events for calibration. $A$ represents the previous flood simulations in 

relationship calibration; (2) Temporal prediction scenario: comparison of calibration and temporal prediction results obtained by selecting different flood events for calibration. $A$ represents the previous calibration and temporal prediction results obtained by selecting the former six floods for relationship calibration in Subsection 4.1, and $B$ represents those obtained by selecting the former three flood events for relationship calibration. (3) Spatial prediction scenario: comparison of spatial prediction results for the whole basin obtained by selecting different sub-basins for calibration. $A$ represents the previous spatial prediction results with relationships calibrated by the discharge data of Qianhancun Station in Subsection 4.1, $B$ represents the spatial prediction results with relationships calibrated by the discharge data of Jurong Station

As shown in Fig. 6 , the $\mathrm{R}^{2}$, NSE, $\mathrm{D}_{\mathrm{p}}$, and $\mathrm{D}_{\mathrm{v}}$ values are located near the 1:1 line. The differences in the four criteria are statistically insignificant according to the $F$-test ( $\alpha=0.05$, Jamshidian et al. 2007), indicating that the use of different flood events for parameter calibration yielded similar results for simulation and temporal prediction. In terms of spatial prediction, the relationships calibrated from different sub-basins could be transferred to the whole basin and generated similar spatial prediction results. These

411 results demonstrate that the impact of flood event selection on model performance is 412 insignificant, indicating that the proposed parameterization scheme of establishing 413 unified relationships between model parameters and driving factors of sub-basins is 414 robust for hydrological modelling in basins with urbanization. Thus, the relationships 415 calibrated based on flood events with the corresponding land-use patterns can be 416 effectively used for flood simulation and prediction under urbanization with certain 417 reliability. 
419 In this study, we proposed an integrated modelling approach to simulate flood events 420 in the QRB, an urbanized basin of south-eastern China. The impacts of imperviousness 421 on runoff generation and runoff routing were both taken into account. Considering the 422 lack of observed data in sub-basins, unified relationships between Nash IUH model 423 parameters and driving factors for all sub-basins were established and calibrated with 424 observed data at the basin outlet. The following conclusions were obtained: (1) the 425 proposed semi-distributed modelling approach can produce reasonable flood simulation 426 results, especially when parameters of the Nash model for any sub-basin are calculated 427 based on calibrated unified relationships between parameters and sub-basin physical 428 characteristics; (2) imperviousness is an important factor that should be considered in 429 flood routing calculations, especially for simulating small or medium floods; (3) the 430 integrated modelling approach is effective, robust and efficient for flood simulation in 431 mesoscale basins and has prediction capability over time and space for future land-use 432 changes and adjacent basins. Future studies need to be carried out to extend the 433 application of the proposed modelling approach to other urbanized basins.

\section{References}

435 Abbaspour KC, Rouholahnejad E, Vaghefi S, Srinivasan R, Yang H, Klove B (2015) A continental-scale hydrology and water quality model for Europe: Calibration and uncertainty of a high-resolution

Arnold CL, Gibbons CJ (1996) Impervious surface coverage - The emergence of a key environmental indicator. Journal of the American Planning Association 62:243-258. https://doi:10.1080/01944369608975688 
Arnold JG, Fohrer N (2005) SWAT2000: current capabilities and research opportunities in applied watershed modelling. Hydrological Processes 19:563-572. https://doi:10.1002/hyp.5611

Bach PM, Rauch W, Mikkelsen PS, McCarthy DT, Deletic A(2014) A critical review of integrated urban water modelling-Urban drainage and beyond Environmental. Modelling \& Software 54:88-107. https://doi:10.1016/j.envsoft.2013.12.018

Bedient PB, Huber WC (1992) Hydrology and Floodplain Analysis. Addison-Wesley, Massachusetts Bian GD, Du JK, Song MM, Xu YP, Xie SP, Zheng WL, Xu CY (2017) A procedure for quantifying runoff response to spatial and temporal changes of impervious surface in Qinhuai River basin of southeastern China. Catena 157:268-278. https://doi:10.1016/j.catena.2017.05.023

Booth DB, Jackson CR (1997) Urbanization of aquatic systems: Degradation thresholds, stormwater detection, and the limits of mitigation. Journal of the American Water Resources Association 33:1077-1090. https://doi:10.1111/j.1752-1688.1997.tb04126.x

Braud I et al. (2013) Evidence of the impact of urbanization on the hydrological regime of a mediumsized periurban catchment in France. Journal of Hydrology 485:5-23. https://doi:10.1016/j.jhydrol.2012.04.049

Burns D, Vitvar T, McDonnell J, Hassett J, Duncan J, Kendall C (2005) Effects of suburban development on runoff generation in the Croton River basin, New York, USA. Journal of Hydrology 311:266281. https://doi:10.1016/j.jhydrol.2005.01.022

Cheng WH, Wang CH, Zhu Y (2006) Taihu Basin Model. Hohai University Press, Nanjing (in chinese)

Deng P, Li ZJ, Xie F (2009) Application of TOPMODEL in Buliu River catchment, Pearl River basin and comparison with Xin'anjiang model. Journal of Lake Sciences 21(3):441-444. (in chinese)

Du JK, Zheng DP, Xu YP, Hu SF, Xu CY (2016) Evaluating functions of reservoirs' storage capacities and locations on daily peak attenuation for Ganjiang River basin using Xinanjiang model. Chinese Geographical Science 26:789-802. https://doi:10.1007/s11769-016-0838-6

Du JK et al. (2013) Hydrological simulation by SWAT model with fixed and varied parameterization approaches under land use change. Water Resources Management 27:2823-2838. https://doi:10.1007/s11269-013-0317-0

Hu HB (2016) Rainstorm flash flood risk assessment using genetic programming: A case study of risk zoning in Beijing. Natural Hazards 83:485-500. https://doi:10.1007/s11069-016-2325-x

Hundecha Y, Bardossy A (2004) Modeling of the effect of land use changes on the runoff generation of 
a river basin through parameter regionalization of a watershed model. Journal of Hydrology 292:281-295. https://doi:10.1016/j.jhydrol.2004.01.002

Jacobson CR (2011) Identification and quantification of the hydrological impacts of imperviousness in urban catchments: A review. Journal of Environmental Management 92:1438-1448. https://doi:10.1016/j.jenvman.2011.01.018

Jamshidian M, Jennrich RI, Liu W (2007) A study of partial F tests for multiple linear regression models. Computational Statistics

$\&$

Data

Analysis

$51: 6269-6284$. https://doi:10.1016/j.csda.2007.01.015

Kaspersen PS, Ravn NH, Arnbjerg-Nielsen K, Madsen H, Drews M (2015) Influence of urban land cover changes and climate change for the exposure of European cities to flooding during highintensity precipitation. In: Rogger $M$ et al. (eds) Changes in Flood Risk and Perception in

Lee JG, Heaney JP (2003) Estimation of urban imperviousness and its impacts on storm water systems. https://doi:10.1061/(asce)0733-9496(2003)129:5(419)

Lhomme J, Bouvier C, Perrin JL (2004) Applying a GIS-based geomorphological routing model in urban catchments. Journal of Hydrology 299:203-216. https://doi:10.1016/j.jhydrol.2004.08.006

Li DC, Qu SM, Shi P, Chen XQ, Xue F, Gou JF, Zhang WH (2018) Development and integration of subdaily flood modelling capability within the SWAT model and a comparison with XAJ model. Water 10. https://doi:10.3390/w10091263

Lin GY, Luo S, Shi YD, Gao Y (2011) Modification and verification of rice field runoff model under non-saturated soil condition. Water Saving Irrigation 10:33-36. (in chinese)

McColl C, Aggett G (2007) Land-use forecasting and hydrologic model integration for improved landuse decision support. Journal of Environmental Management 84:494-512. https://doi:10.1016/j.jenvman.2006.06.023

McCuen RH, Knight Z, Cutter AG (2006) Evaluation of the Nash-Sutcliffe efficiency index. Journal of Hydrologic Engineering 11:597-602. https://doi:10.1061/(asce)1084-0699(2006)11:6(597) 
Nash JE (1960) A unit hydrograph study with particular reference to British catchments. Proc Inst Civ Eng, London 17:249-282

Oudin L, Salavati B, Furusho-Percot C, Ribstein P, Saadi M (2018) Hydrological impacts of urbanization at the catchment scale. Journal of Hydrology 559:774-786. https://doi:10.1016/j.jhydrol.2018.02.064

Patra S, Sahoo S, Mishra P, Mahapatra SC (2018) Impacts of urbanization on land use/cover changes and its probable implications on local climate and groundwater level. Journal of Urban Management

Praskievicz S, Chang H (2009) A review of hydrological modelling of basin-scale climate change and 7:70-84. https://doi:10.1016/j.jum.2018.04.006 urban development impacts. Progress in Physical Geography 33:650-671. https://doi:10.1177/0309133309348098

514 Refsgaard JC (1997) Parameterisation, calibration and validation of distributed hydrological models. Journal of Hydrology 198:69-97. https://doi:10.1016/s0022-1694(96)03329-x

Rodriguez F, Andrieu H, Morena F (2008) A distributed hydrological model for urbanized areas - Model development and application to case studies. Journal of Hydrology 351:268-287. https://doi:10.1016/j.jhydrol.2007.12.007

Rodriguez JJ, Kuncheva LI, Alonso CJ (2006) Rotation Forest: A New Classifier Ensemble Method.

Salvadore E, Bronders J, Batelaan O (2015) Hydrological modelling of urbanized catchments: A review and future directions. Journal of Hydrology 529:62-81. https://doi:10.1016/j.jhydrol.2015.06.028

Schueler TR, Fraley-McNeal L, Cappiella K (2009) Is impervious cover still important? Review of recent research. Journal of Hydrologic Engineering 14:309-315. https://doi:10.1061/(asce)10840699(2009)14:4(309)

Trinh DH, Chui TFM (2013) Assessing the hydrologic restoration of an urbanized area via an integrated distributed hydrological model. Hydrology and Earth System Sciences 17:4789-4801.

531 USACE-HEC (2000) Hydrologic modeling system HEC-HMS, Technical Reference Manual. US Army 
533 Valeo C, Moin SMA (2000) Grid-resolution effects on a model for integrating urban and rural areas. 1085(20001015)14:14<2505::AID-HYP111>3.0.CO;2-3

$536 \mathrm{Xu}$ C-Y (1999) Estimation of parameters of a conceptual water balance model for ungauged catchments.

$538 \mathrm{Xu}$ C-Y (2003) Testing the transferability of regression equations derived from small subcatchments to https://doi:10.5194/hess-7-317-2003

541 Yang X, Magnusson J, Rizzi J, Xu C-Y (2018) Runoff prediction in ungauged catchments in Norway: comparison of regionalization approaches. Hydrology Research 49:487-505. https://doi:10.2166/nh.2017.071

544 Zhang Y et al. (2018) Simulation and assessment of urbanization impacts on runoff metrics: Insights from landuse changes. Journal of Hydrology 560:247-258. https://doi:10.1016/j.jhydrol.2018.03.031

547 Zhao RJ (1992) The Xinanjiang model applied in China. Journal of Hydrology 135(1):371381.https://doi.org/10.1016/0022-1694(92)90096-E 